\title{
Imaging coherent electron wave flow in a two-dimensional electron gas
}

\author{
B.J. LeRoy ${ }^{\mathrm{a}, 1}$, A.C. Bleszynski ${ }^{\mathrm{a}}$, M.A. Topinka ${ }^{\mathrm{b}}$, R.M. Westervelt ${ }^{\mathrm{a}, \mathrm{b}}{ }^{\text {, S.E.J. Shaw }}{ }^{\mathrm{a}}$, \\ E.J. Heller ${ }^{\mathrm{a}, \mathrm{c}}$, K.D. Maranowski ${ }^{\mathrm{d}}$, A.C. Gossard ${ }^{\mathrm{d}}$ \\ ${ }^{a}$ Department of Physics, Harvard University, Cambridge, MA 02138 USA \\ b Division of Engineering and Applied Sciences, Harvard University, Cambridge, MA 02138 USA \\ ${ }^{\mathrm{c}}$ Department of Chemistry and Chemical Biology, Harvard University, Cambridge, MA 02138 USA \\ ${ }^{\mathrm{d}}$ Materials Department, University of California, Santa Barbara, CA 93106 USA
}

\begin{abstract}
We measure the energy distribution of electrons passing through a two-dimensional electron gas using a scanning probe microscope. We present direct spatial images of coherent electron wave flow from a quantum point contact formed in a GaAs/AlGaAs two-dimensional electron gas using a liquid He cooled SPM. A negative voltage is placed on the tip, which creates a small region of depleted electrons that backscatters electron waves. Oscillating the voltage on the tip and locking into this frequency gives the spatial derivative of electron flow perpendicular to the direction of current flow. We show images of electron flow using this method. By measuring the amount of electrons backscattered as a function of the voltage applied to the tip, the energy distribution of electrons is measured.
\end{abstract}

Key words: Quantum Point Contact; Scanning Probe Microscopy; Two-dimesional electron gas;

Scanning probe microscopy (SPM) has become increasingly important for understanding mescoscopic phenomena including quantum point contacts, the quantum Hall effect and carbon nanotubes [1-6]. SPM techniques provide information on a local scale, which is not available from bulk transport measurements. In this paper, we present images showing coherent electron flow from a quantum point contact (QPC). We demonstrate a technique for imaging the spatial derivative of electron flow perpendicular to the direction of current flow by oscillating the voltage on the tip and locking into this frequency. We also measure the distribution of electrons as a function of energy by measuring the number of electrons backscattered as a function of the voltage applied to the tip.

Fig. 1(A) shows the measurement setup used to image coherent electron wave flow. The sample used is a GaAs/AlGaAs heterostructure with a two-dimensional

\footnotetext{
1 Corresponding author. E-mail: leroy@physics.harvard.edu
}

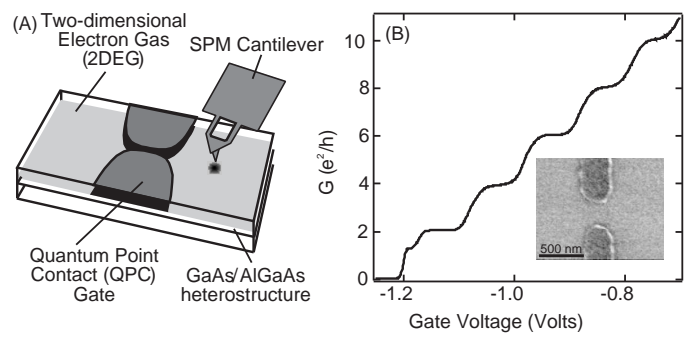

Fig. 1. (A) Schematic diagram of the measurement setup used to image electron flow. (B) Quantum point contact conductance versus gate voltage showing conductance plateaus. The inset is an SEM picture of the device.

electron gas (2DEG) located $57 \mathrm{~nm}$ beneath the surface. Metal gates are deposited on the surface to form the QPC. A negative voltage is put on the SPM tip with respect to the $2 \mathrm{DEG}$, which creates a small depleted region directly below the tip. The depleted region can backscatter electrons reducing the conductance through the QPC. By raster scanning the tip 

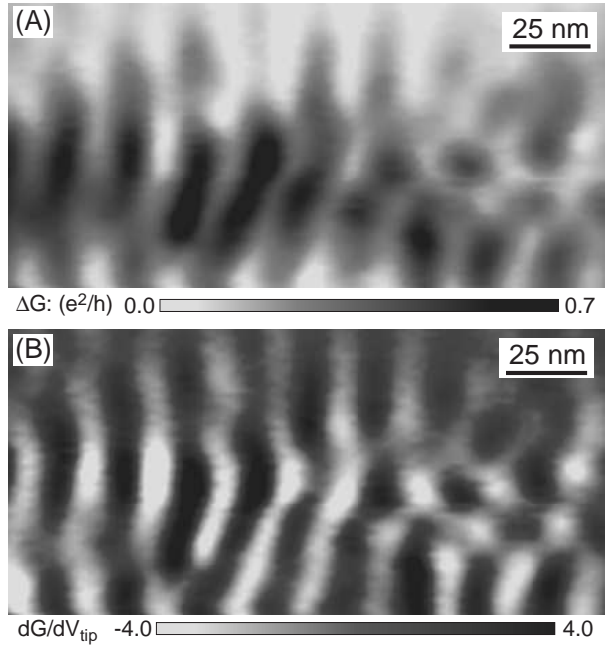

Fig. 2. (A) Image of electron flow taken with a constant DC voltage on the tip. Light areas are areas of high electron flow and dark areas have little or no electron flow. (B) Image of the differential conductance with respect to tip voltage taken at the same location. This images the spatial derivative of electron flow.

over the sample and recording the conductance, an image of electron flow is obtained. Fig. 1(B) is a plot of the QPC conductance $\mathrm{G}$, versus its width controlled by the voltage on the gate. Well defined conductance plateaus are visible at multiples of $2 \mathrm{e}^{2} / \mathrm{h}[7]$. The inset shows an SEM micrograph of the device.

Fig. 2 shows images of electron flow taken simultaneously with the two different measurement techniques. Fig. 2(A) is an image of how the conductance, $\mathrm{G}$ changes as a function of tip position. Areas of high $\Delta \mathrm{G}$ correspond to areas of high electron flow $[1,2]$. Fig. $2(\mathrm{~B})$ is a spatial derivative of Fig. 2(A) acquired by adding a small oscillating voltage to the DC voltage applied to the tip and locking into that frequency. Oscillating the tip voltage changes the size of the depleted region below the tip and hence the location where electrons are backscattered. This technique therefore gives the spatial derivative of the electron flow because we are only sensitive to signals that change as a function of tip position.

Fig. 3 shows how we can use the tip voltage to probe the distribution of electrons in the 2DEG. The tip reduces the conductance through the QPC only when the voltage on it is sufficient to deplete the electrons directly below and backscatter them[8]. There is a distribution of electron energies impingent on the tip, requiring a different tip voltage to backscatter the different energy electrons. Fig. 3(A) shows the tip potential for three different tip voltages. As the voltage increases, the tip backscatters electrons of increasing energy. For the lowest and highest tip potential, there is little change in the conductance with tip voltage since
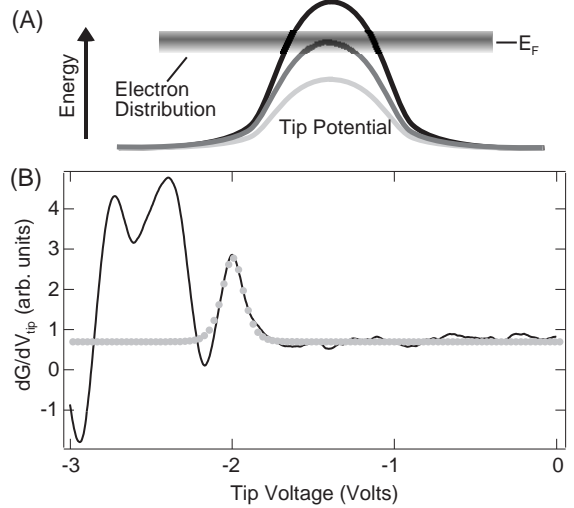

Fig. 3. (A) Shows the effect of tip voltage on the backscattering of electrons. The bottom and top curve have little change in backscattering with tip voltage. For the middle curve, the tip potential is peaked at the Fermi energy giving a large change in conductance with tip voltage. (B) Measurement of the differential conductance with respect to tip voltage as a function of tip voltage. The curve near -2 Volts measures the energy distribution of the electrons. The gray circles are the expected thermal distribution of electrons, which agrees very well with the measurement.

the distribution of electrons is not changing quickly. In contrast, when the tip potential is at the Fermi energy there is the largest change in conductance with tip voltage since there is a largest change in the electron distribution at this energy. Fig. 3(B) shows the differential conductance with respect to tip voltage as a function of tip voltage. For a thermal distribution of electrons, we would expect the signal to be the derivative of the Fermi function with respect to energy. This is shown by the gray circles in Fig. 3(B) which fit the data very well, indicating that we are measuring the distribution of electrons. The oscillations in the signal for larger tip voltages come from the size of the depleted region under the tip growing and the position of the backscattered electrons changing. The tip is just moving through the interference fringes seen in Fig. 2.

\section{References}

[1] M.A. Topinka et al., Science 289, 2323 (2000).

[2] M.A. Topinka et al., Nature 410, 183 (2001).

[3] B.J. LeRoy et al., APL 80, 4431 (2002).

[4] R. Crook et al., J. Phys. Condens. Matter 12, L735 (2000).

[5] G. Finkelstein et al., Science 289, 90 (2000).

[6] M.T. Woodside, P.L. McEuen, Science 296, 1098 (2002).

[7] B.J. van Wees et al., PRL 60, 848 (1988)., D. A. Wharam et al., J. Phys. C Solid State Phys. 21, 209 (1988).

[8] M.A. Topinka et al., to be published. 